\title{
Activity-driven topological glass
}

\section{Active topological glass}

Authors: Jan Smrek, Iurii Chubak, Christos N. Likos, and Kurt Kremer

Nature Communications, 11: 26, 2020.

doi.org/10.1038/s41467-019-13696-z

\section{Emergence of active topological glass through directed chain dynamics and} nonequilibrium phase separation

Authors: Iurii Chubak, Christos N. Likos, Kurt Kremer, and Jan Smrek

Physical Review Research, 2: 043249, 2020.

doi.org/10.1103/PhysRevResearch.2.043249

Recommended with a Commentary by Dimitris Vlassopoulos, Department of Materials Science \& Technology, University of Crete and Institute of Electronic Structure \& Laser, FORTH, Greece

It is well known that particle crowding due to the increased packing fractions in colloidal suspensions leads to glass formation. However this is not the case for linear or branched polymers. On the other hand, recently, it was shown by means of simulations that a new type of topological glass can be formed with non-concatenated ring polymers at large concentrations, if a small fraction of the rings is immobilized (this is the pinning process in simulations) [1]. From the viewpoint of an experimentalist, this concept of topological glass is intriguing, however materializing the partial immobilization of the sample is not straightforward. The papers of Smrek and co-workers show how to circumvent this difficulty and, more importantly, open a new direction in the field: activity promotes glass formation. The reason is its coupling to the loopy topology of the rings at high concentrations (clearly above their overlap) which enhances ring-ring threading sufficiently so that the relaxation of the rings takes place cooperatively at extremely long relaxation times. This is distinct from the conventional active glass where activity promotes fluidity. This exciting result has many consequences and poses interesting questions, which we outline below:

The mutual interpenetration of ring polymers was conjectured in rheological studies of (experimentally) pure entangled rings and confirmed by MD simulations under both equilibrium 
and flow conditions [2,3]. What is not clear is the extent of threading needed to affect ring dynamics and whether each ring should contain an active block or only a fraction. A detailed study addressing these issues will be beneficial not only in addressing the vitrification of rings (and other loopy structures) in solution [4] but also elucidating the physics underlying the presence of slow modes in the stress relaxation of ring melts. In this context, the current state-ofthe-art model for stress relaxation of entangled rings [5] does not account for their mutual threading as a delaying factor. A recent alternative approach to account for the slow mode considers rings as effective soft (fuzzy) particles and threading enters implicitly via the structure factor [6]. If indeed such a topological glass is observed in melts of rings, rings would constitute a paradigm exhibiting two glass transitions at different scales, the conventional polymer glass, associated to restrictions at segmental level, and the topological glass, due to the interpenetration of loops. It should be noted that the active topological glass exhibits a smoother non-ergodicity transition compared to conventional glasses.

The use of activity as a means to slow-down or even arrest macroscopic motion offers interesting possibilities. Here, the authors discussed the formation of topological constraints. One may think of the potential to reduce defects and promote order in topologically heterogeneous materials. A more delicate point is how to link the activity with the pinning of [1], since in both cases the end result is threading and promotion of the glass. Actually, a challenge to address is the role of very large molar mass and in particular whether in this limit the enhanced multiple threading may yield pinning and glass formation.

The active part which promotes threading is stretched and quantifying the correlation between hot/cold temperature and stiff/flexible ring segments (corresponding to active/passive segments) would help to better understand and control the impact of activity. In fact, the authors have examined the interplay between nonequilibrium phase separation (active-passive) and glass formation, and found that it relates to the different segmental dynamics. In this context, a systematic study of the effect of varying length and fraction of active block, as well as the total molar mass, would be particularly helpful.

The simulated picture of threaded rings is reminiscent of the gyroid phase in block copolymers, and this is an interesting analogy to explore, bearing in mind that upon threading there is no thermodynamic equilibrium.

In closing this brief commentary, I wish to share a few simple thoughts about experimental materialization of active topological glass. Block copolymer rings can be synthesized and are available. However, the different mobility and stiffness of the blocks is likely not sufficient to promote or enhance threading as the fluctuations should go beyond thermal noise. In addition, microphase separation may complicate the situation. One may get inspiration from active particles (for example, Janus) and consider for example poly(ethylene oxide) rings having a block coated with platinum; on adding hydrogen peroxide in water, the coated block becomes active. However, this approach does not provide reversibility. Alternatively, diblock copolymer rings having a short magnetically active block (say, based on ferrocene) could become active/passive on switching on/off a magnetic field. This may prove useful in networks with loops, both in bulk and under confinement, which can exhibit liquid-like and solid-like response 
with enhanced mechanical properties, at wish. Of course there even in the absence of field the magnetic block will likely aggregate, so this poses another challenge to address. If successful, such approach could open the route to explore active tadpoles, i.e., passive rings with active grafts which shall drive threading, offering another possibility for topological glass.

Finally, there is also the question of biological relevance, given the loopy packing of chromosome territories. Well, reading this interesting paper one can find answers and suggestions for further studies in this context.

\section{References}

[1] D. Michieletto and M. E. Turner, A topologically driven glass in ring polymers, PNAS, 113(19): 5195-5200, 2016. doi.org/10.1073/pnas.1520665113.

[2] D. G. Tsalikis, V. G. Mavratnzas, and D. Vlassopoulos, Analysis of Slow Modes in Ring Polymers: Threading of Rings Controls Long-Time Relaxation, ACS Macro Lett., 5: 755-760, 2016. doi.org/10.1021/acsmacrolett.6b00259.

[3] T. C. O'Connor, T. Ge, M. Rubinstein, and G. S. Grest, Topological Linking Drives Anomalous Thickening of Ring Polymers in Weak Extensional Flows, Phys. Rev. Lett. 124: 027801, 2020. doi.org/10.1103/PhysRevLett.124.027801.

[4] D. Michieletto, N. Nahali, and A. Rosa, Glassiness and Heterogeneous Dynamics in Dense Solutions of Ring Polymers, Phys. Rev. Lett. 119: 197801, 2017. doi.org/10.1103/PhysRevLett.119.197801

[5] T. Ge, S. Panyukov, and M. Rubinstein, Self-similar conformations and dynamics in entangled melts and solutions of nonconcatenated ring polymers, Macromolecules 49: 708-722, 2016. doi.org/10.1021/acs.macromol.5b02319.

[6] B. Mei, Z. E. Dell, and K. S. Schweizer, Theory of Transient Localization, Activated Dynamics, and a Macromolecular Glass Transition in Ring Polymer Liquids, ACS Macro Lett., 10: 1229-1235, 2021. doi.org/10.1021/acsmacrolett. $1 \mathrm{c} 00530$. 